\title{
Integration of Primary Care and Psychiatry: A New Paradigm for Medical Student Clerkships
}

\author{
Kirsten M. Wilkins, $\mathrm{MD}^{7}$, Ada M. Fenick, MD², Matthew N. Goldenberg, MD, MSc ${ }^{7}$, \\ Peter J. Ellis, $M D, M P H^{3}$, Andres Barkil-Oteo, $M D^{7}$, and Robert M. Rohrbaugh, $\mathrm{MD}^{1}$ \\ 'Department of Psychiatry, Yale University School of Medicine, New Haven, CT, USA; ${ }^{2}$ Department of Pediatrics, Yale University School of Medicine, \\ New Haven, CT, USA; ${ }^{3}$ Department of Medicine, Yale University School of Medicine, New Haven, CT, USA.
}

BACKGROUND: Public health crises in primary care and psychiatry have prompted development of innovative, integrated care models, yet undergraduate medical education is not currently designed to prepare future physicians to work within such systems.

AIM:To implement an integrated primary care-psychiatry clerkship for third-year medical students.

SETTING: Undergraduate medical education, amid institutional curriculum reform.

PARTICIPANTS: Two hundred thirty-seven medical students participated in the clerkship in academic years 2015-2017.

PROGRAM DESCRIPTION: Educators in psychiatry, internal medicine, and pediatrics developed a 12 -week integrated Biopsychosocial Approach to Health (BAH)/Primary Care-Psychiatry Clerkship. The clerkship provides students clinical experience in primary care, psychiatry, and integrated care settings, and a longitudinal, integrated didactic series covering key areas of interface between the two disciplines.

PROGRAM EVALUATION: Students reported satisfaction with the clerkship overall, rating it 3.9-4.3 on a 1-5 Likert scale, but many found its clinical curriculum and administrative organization disorienting. Students appreciated the conceptual rationale integrating primary care and psychiatry more in the classroom setting than in the clinical setting.

CONCLUSIONS: While preliminary clerkship outcomes are promising, further optimization and evaluation of clinical and classroom curricula are ongoing. This novel educational paradigm is one model for preparing students for the integrated healthcare system of the twenty-first century.

KEY WORDS: medical student and residency education; medical education: curriculum development/evaluation; primary care; mental health.

J Gen Intern Med 33(1):120-4

DOI: $10.1007 / \mathrm{s} 11606-017-4169-9$

(c) Society of General Internal Medicine 2017

Received February 27, 2017

Revised June 16, 2017

Accepted August 16, 2017

Published online August 28, 2017

\section{INTRODUCTION}

Two related major public health crises have emerged at the interface of primary care and psychiatry. The first is the challenge of providing care for the epidemic of mental health and substance use disorders in the patient population served by primary care clinics. Mental health and substance use disorders are the leading global cause of all non-fatal burden of disease, and much of the burden for addressing these disorders falls to non-mental health specialists, including primary care and other physicians. ${ }^{1}$ The second crisis is that patients with serious mental illness and substance use disorders die 1520 years earlier than patients without mental illness ${ }^{2}$; lack of access to basic primary care services for these patients may contribute to this striking and tragic health disparity. ${ }^{3-5}$

Both crises have illuminated the paucity of integrated, interprofessional models for delivering primary care and mental health services. The past decade has witnessed research on new models of integrated care that have been effective, ${ }^{6}$ the largest of which resides within the Veterans Affairs Healthcare System. ${ }^{7}$ Dissemination of these new models was promoted by passage of the Affordable Care Act. ${ }^{8}$ However, adoption is still slow, especially in academic medical centers. ${ }^{9}$ Widespread dissemination of these new models requires the development of a workforce with knowledge, skills, and positive attitudes toward integrated care. A few combined medicinepsychiatry residency programs have been developed, ${ }^{10}$ but in order to have an adequate pipeline of health professionals interested in and capable of mastering this challenge, training should ideally begin in medical school.

Meanwhile, undergraduate medical education has undergone significant reform as schools aim to prepare medical students for healthcare in the twenty-first century. ${ }^{11}$ Newly integrated course and clerkship curricula offer unique opportunities for cross-departmental collaboration and dissolution of longstanding silos. Frequently, when disciplines are paired for integrated clerkships, psychiatry is paired with neurology. Indeed, a persuasive argument has been made for their integration in medical education. ${ }^{12}$ An equally compelling case can be made for the integration of psychiatry and primary care. These two disciplines share important values, including a biopsychosocial approach to patient care, the management of chronic conditions through a focused relationship between a 
doctor and patient, and counseling for improved function. A pairing of psychiatry and primary care also provides an opportunity to demonstrate a population-based approach to screening, diagnosing, and treating mental health disorders.

\section{SETTING AND PARTICIPANTS}

We developed an integrated Biopsychosocial Approach to Health/Primary Care-Psychiatry (BAH) clerkship at the Yale University School of Medicine. This clerkship emerged from a school-wide curriculum redesign that began in 2012, and resulted in a clerkship year with four 12-week integrated clerkships. Discipline-based clerkships are paired according to shared themes (e.g., similar approaches to patient care, common clinical conditions in individuals and populations). The BAH clerkship developed from collaboration among the psychiatry, internal medicine, and pediatrics departments. Core clerkship themes include health promotion and disease prevention, population health, social determinants of health, behavioral change, systems-based care, and management of chronic disease. To design the clerkship, we formed a steering committee of interdisciplinary faculty, residents, students, and staff. The committee determined the competencies expected of learners at the time of clerkship completion and a series of outcome-based objectives. The steering committee focused on the integration of complex skills, knowledge, and attitudes required for the practice of medicine in the twenty-first century.

This study was deemed exempt by the Yale Institutional Review Board.

\section{PROGRAM DESCRIPTION}

\section{Clinical Curriculum}

Clerkship leaders sought to expose students to the core knowledge, skills, and attitudes of both primary care and psychiatry. In the psychiatry rotation, students rotate in inpatient, outpatient, and emergency or consult-liaison settings. In the primary care rotation, students rotate in adult and pediatric clinics. An additional goal was to expose students to integrated care clinics where professionals from the two disciplines work together. For example, at a primary care site within the VA Connecticut Healthcare System, students rotated with embedded psychiatrists who collaborate closely with primary care physicians in the care of veterans with comorbid medical and psychiatric illnesses. Other students rotated at a local methadone program where patients with opioid use disorder receive both treatment for substance use and primary care. In these settings, students received critical education in the complex needs of underserved populations and systems of care in their own community. We endeavored to assign each student in the BAH clerkship to a longitudinal integrated care clinic for an 8week experience; assignments were made by convenience.

\section{Didactic Curriculum}

We employed a wide array of instructional methods that were learner-centered and aligned with clerkship content and desired outcomes. All students attend a "precede" of didactics on day 1 of the clerkship to address content and core skills which are applicable to both disciplines (e.g., rationale for the integrated clerkship, introduction to the biopsychosocial model, epidemiology of local healthcare and population, and mental status and physical exam skills training). All students come together once each week throughout the 12-week clerkship for an integrated didactic series. This series covers key areas of clinical interface between primary care and psychiatry and employs a variety of interactive pedagogies aimed at teaching key content and promoting student teamwork (Appendix Table 2). Topics for this integrated series were carefully chosen by clerkship directors, who prioritized common mental health-related concerns with which patients present in primary care settings. ${ }^{13}$ Additional primary care didactic sessions address topics such as hypertension, diabetes, and social determinants of health. Additional didactic sessions in psychiatry address topics including mood disorders, psychotic disorders, sexual health, and insomnia.

The clerkship ends with a 1-day integrated "postcede" of activities designed to provide comprehensive formative assessment, feedback, and reflection. Students rotate through several stations at the Yale Center for Medical Simulation that use standardized patients, manikin-based simulation, and videos to allow students to demonstrate skills in motivational interviewing, mental status exam, differential diagnosis, and clinical reasoning. All clerkship students participate in a wrap-up session that encourages reflection on themes and experiences of interdisciplinary integration over the previous 12 weeks. Finally, students meet with clerkship directors to provide mutual feedback in order to promote the concept of lifelong learning and self-improvement.

\section{Student Assessment}

Students are provided formative assessment by faculty and resident supervisors throughout the clerkship. All students keep a $\log$ to track their overall clinical encounters, directly observed encounters, mid-clerkship feedback, and other required activities. Additionally, for 6 weeks, students are assigned a faculty or senior resident tutor to help them hone interview and writing skills and to encourage explicit consideration of the biopsychosocial aspects of health and self-reflection. As above, the clerkship "postcede" includes activities designed to provide formative assessment of skills obtained during the 12-week clerkship. In addition, all students complete a multiplechoice knowledge examination. A student's final clerkship grade is determined by numerical and narrative clinical evaluations from faculty and resident preceptors. 
Table 1 Integrated Biopsychosocial Approach to Health (BAH) Clerkship: Student Feedback* and Subsequent Revisions after Year 1

\begin{tabular}{|c|c|c|}
\hline Feedback Theme & Sample Student Comments & Revisions Made \\
\hline $\begin{array}{l}\text { Lack of appreciation for conceptual } \\
\text { integration of primary care } \\
\text { and psychiatry }\end{array}$ & $\begin{array}{l}\text { "There was no bridge [between the two disciplines]." } \\
\text { "I did not see a lot of integration, in part because I } \\
\text { actively tried to compartmentalize the two." }\end{array}$ & $\begin{array}{l}\text { Added more explicit discussion of rationale } \\
\text { for integrated clerkship (i.e., teaching about } \\
\text { the biopsychosocial approach on day 1) } \\
\text { Added the weekly "Top Ten" didactic series to } \\
\text { explicitly cover areas of clinical interface } \\
\text { between the two disciplines } \\
\text { Provided faculty development for preceptors } \\
\text { regarding clerkship rationale and encouraged } \\
\text { them to emphasize integration with students }\end{array}$ \\
\hline $\begin{array}{l}\text { Lack of administrative } \\
\text { organization }\end{array}$ & $\begin{array}{l}\text { "[Clerkship organization] was extremely confusing. } \\
\text { I wish the clerkships had just been separate." } \\
\text { "It became confusing to keep track of what we had } \\
\text { to do for one [clerkship component] versus the } \\
\text { other as we got emails from two separate people } \\
\text { instead of having one central administrator." }\end{array}$ & $\begin{array}{l}\text { Changed structure to } 6 \text { consecutive weeks of } \\
\text { primary care and } 6 \text { consecutive weeks of } \\
\text { psychiatry (see Fig. 1) } \\
\text { Improved delineation of administrative } \\
\text { responsibility for each component }\end{array}$ \\
\hline
\end{tabular}

Challenges of the "mixed month" $\dagger$

Value of integrated primary carepsychiatry clinic experiences

Appreciation of the clinical interface between primary care and psychiatry
"There was too much switching around...the schedule was very fragmented."

"Try not to 'integrate' primary care and psychiatry so much...that's where things get confusing."

"My continuity clinic integrated the two [disciplines] in an exemplary way: providing primary care to a population comprised largely of patients with substance use disorders and very often comorbid psychiatric disorders."

"Most of the patients in my primary care office presented with psychiatric issues...it was helpful that I was thinking about psych in parallel."

"It was clear that many patients' psychiatric problems are also affecting or affected by their other somatic health problems, so an awareness of how those are linked was very helpful."
Discontinued the "mixed month"

Separated primary care and psychiatry didactics except for the "Top Ten"

Attempted to assign each student to an integrated clinic whenever possible, limited by local availability of integrated sites

Increased emphasis on areas of clinical interface in the "Top Ten" didactic series

\section{PROGRAM EVALUATION}

The BAH clerkship began in June 2015, and in academic year 2015-2016, 95 students (100\% of class) completed the clerkship. We evaluated the program via our institution's traditional means: anonymous online evaluations, student feedback sessions with clerkship directors, and student advisory groups with the school's associate director of curriculum assessment. Students used Likert scales to numerically rate $(1-5$, where $1=$ poor and $5=$ excellent) each clerkship component overall, as well as in specific areas such as patient care, clinical teaching, and assessment. During academic year 2015-2016, the clerkship was moderately to very well received: the primary care and psychiatry components received average overall ratings of 3.9 and 4.2, respectively. Comments from students revealed areas in which they appreciated the clinical interface between primary care and psychiatry, as well as the challenges they faced in conceptually bridging the disciplines (Table 1).

A key lesson learned in implementing the new clerkship is that "clerkship integration" is much more easily achieved in the classroom than in the clinical setting. In the initial iteration of the clerkship, we piloted a mix of clinical experiences in both disciplines as well as integrated care settings throughout the 12 weeks (Fig. 1). Despite our sense that the care models of psychiatry and primary care share important values, student feedback indicated that this mixed structure was disorienting to clinically naïve students, who voiced a preference for clearly distinct clinical experiences. As the clerkship has evolved, we have responded to student feedback by organizing primary care and psychiatry clinical experiences separately, while strengthening the integrated classroom curriculum (Fig. 1). Clerkship evaluation data from academic year 2016-2017 ( $n=142$ students) reveal improved overall Likert scale ratings of both primary care (4.0) and psychiatry (4.3). The clerkship still offers exposure to integrated clinics during the outpatient psychiatry component, but we find that availability of integrated sites is not yet sufficient to meet the demands of new educational models such as the BAH clerkship. Approximately $50-60 \%$ of clerkship students can be assigned to an integrated care clinic; due to limited availability of integrated sites, the remainder are assigned to a psychiatry clinic for their continuity experience (e.g., private practice, child, community).

\section{DISCUSSION}

Integrated curricula are increasingly common in undergraduate medical education. ${ }^{14}$ In recent years, several medical schools have introduced longitudinal integrated clerkships, 


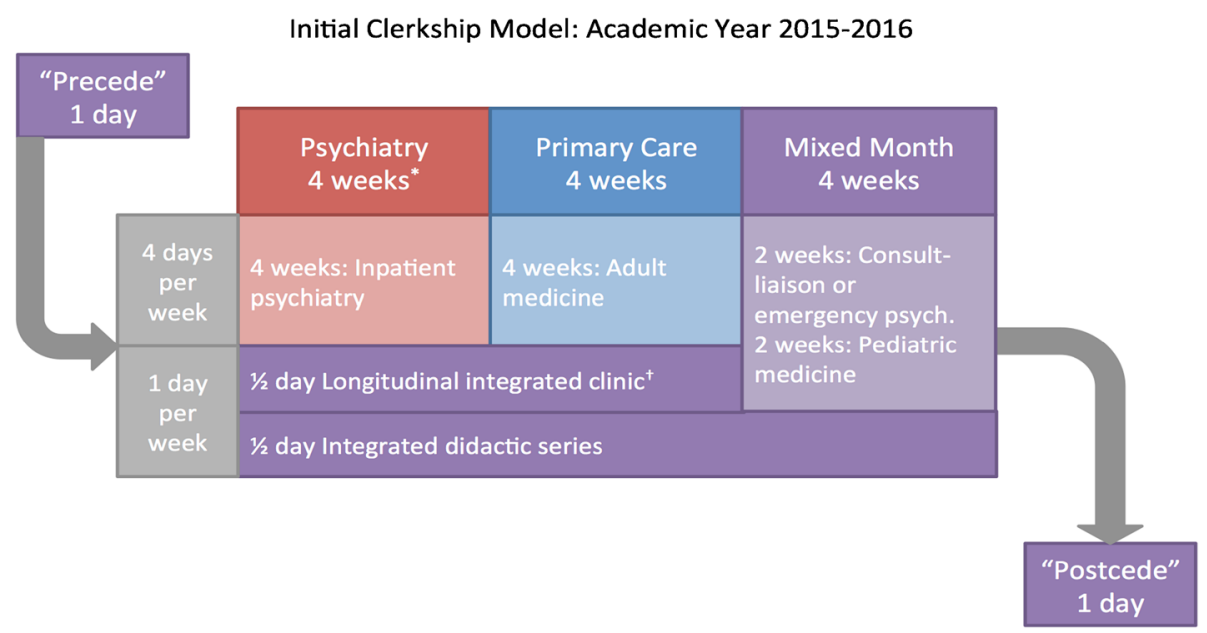

Current Clerkship Model: Academic Year 2016-2017

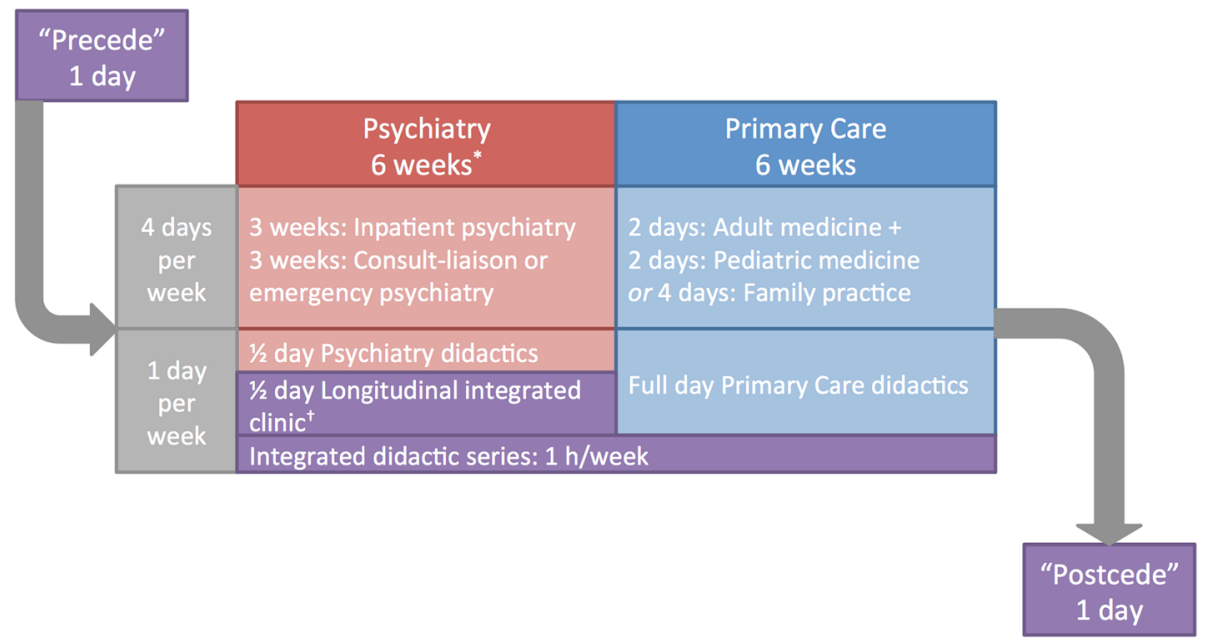

Figure 1 Biopsychosocial approach to health clerkship: initial model and current model. *Half of the clerkship students begin in psychiatry; half begin in primary care. ${ }^{\dagger}$ Students are assigned to an integrated care clinic or, if one is not available, to an outpatient psychiatry clinic.

which integrate multiple clerkships throughout the year and are offered to a portion of the medical school class during the core clinical year. ${ }^{15-17}$ To our knowledge, this is the first integrated 12 -week primary care-psychiatry clerkship required of all students at a US medical school.

In our initial design, we ambitiously aimed to provide students a "mixed month" of primary care and psychiatry clinical experiences as well as a longitudinal clinical experience. Students were largely dissatisfied with this model, which they found disorienting. It may be that conceptual integration of primary care and psychiatry is better appreciated by the advanced student who has already experienced clinical immersion in each singular discipline or who is able to actually experience an integrated care site. Feedback from students in academic year 2016-2017 suggests that they are more satisfied with the new model that provides more distinct clinical focus in each discipline.

Despite our efforts to highlight the multiple areas of overlap between primary care and psychiatry, some students commented that they appreciated no integration between the two. It may be that a student's appreciation of integration is partially dependent on the delivery of care at specific sites or by specific providers. Students who rotate in integrated care clinics report those sites to be highly effective in conveying the relevance of the integrated clerkship, although, as noted, we are currently unable to place all students in an integrated care site. A lack of available integrated care clerkship sites is consistent with findings from a recent survey of psychiatry clerkship directors. ${ }^{18}$ While we have developed didactics and simulated integrated experiences, the identification and recruitment of high-quality integrated care sites remains a top priority for clerkship leaders.

While innovative, this clerkship model has been implemented at only one school and has been in existence for only 2 years; therefore, results are preliminary and may not be generalizable to other schools or student populations. We plan further analysis of student feedback regarding the clerkship's distinct clinical experiences and strengthened integrated classroom curriculum. Additionally, we are designing a pre- and post-clerkship evaluation of students' ability to evaluate and treat patients using a biopsychosocial framework. We are 
interested in faculty response to this new model, especially whether the students' focus on primary care-psychiatry integration has any impact on faculty practice patterns, patient care strategies, or self-directed learning.

The integration of primary care and psychiatry into one clinical clerkship experience offers an innovative interdisciplinary educational paradigm with the potential to serve as an international model for teaching medical students to care for patients at this interface. Challenges to implementing this paradigm include developing clinical sites which offer an integrated experience, and the limited ability of students early in their clinical training to apprehend similarities in the two clinical disciplines. While further optimization of the experience is needed, we believe training students in an interdisciplinary framework will increase understanding of and reduce stigma associated with the public health crises facing both primary care and psychiatry.

Corresponding Author: Kirsten M. Wilkins, MD; Department of Psychiatry Yale University School of Medicine, New Haven, CT, USA (e-mail: kirsten.wilkins@yale.edu).

Contributors The authors wish to thank Dr. Richard Belitsky, Dr. Michael Schwartz, Dr. Walter Kernan, and the members of the Biopsychosocial Approach to Health Clerkship Steering Committee for their support and service.

Funders None.

\section{Compliance with Ethical Standards:}

Conflict of Interest: Dr. Wilkins reports honoraria from Oakstone Board Review Course (speaker). All other authors declare that they have no conflict of interest.

\section{REFERENCES}

1. Whiteford HA, Degenhardt L, Rehm J, et al. Global burden of disease attributable to mental and substance use disorders: findings from the Global Burden of Disease Study 2010. Lancet. 2013;382(9904):1575-86.

2. Thornicroft G. Physical health disparities and mental illness: The scandal of premature mortality. Br J Psychiatry. 2011;199(6):441-2.

3. Benjamin-Johnson R, Moore A, Gilmore J, Watkins K. Access to medical care, use of preventive services, and chronic conditions among adults in substance abuse treatment. Psychiatr Serv. 2009;60:1676-9.

4. Kilbourne AM, McCarthy JF, Post EP, et al. Access to and satisfaction with care comparing patients with and without serious mental illness. Int J Psychiatry Med. 2006;36:383-99.

5. Levinson Miller CL, Druss BG, Dombrowski EA, Rosenheck RA Barriers to primary medical care among patients at a community mental health center. Psychiatr Serv. 2003;54:1158-60.

6. Manderscheid R, Kathol R. Fostering sustainable, integrated medical and behavioral health services in medical settings. Ann Intern Med. 2014; 160:61-5.

7. Zeiss AM, Karlin BE. Integrating mental health and primary care services in the Department of Veterans Affairs Health Care System. J Clin Psychol Med Settings. 2008;15:73-8.
8. Croft B, Parish SL. Care integration in the Patient Protection and Affordable Care Act: implications for behavioral health. Adm Policy Ment Health. 2013;40:258-63.

9. Borden WB, Mushlin AI, Gordon JE, Leiman JM, Pardes H. A new conceptual framework for academic health centers. Acad Med. 2015;90:569-73.

10. Doebeling CC, Pitkin AK, Malis R, Yates WR. Combined internal medicine-psychiatry and family medicine-psychiatry training programs, 1999-2000: program directors' perspectives. Acad Med. 2001;76:124752.

11. Irby DM, Cooke M, O'Brien BC. Calls for reform of medical education by the Carnegie Foundation for the Advancement of Teaching: 1910 and 2010. Acad Med. 2010;85:220-7.

12. Reilly TJ. The neurology-psychiatry divide: A thought experiment. BJPsych Bull. 2015;39(3):134-5.

13. Hudson DL, Kaphingst KA, Croston MA, Blanchard MS, Goodman MS. Estimates of mental health problems in a vulnerable population within a primary care setting. J Health Care Poor Underserved. 2016;27(1):308-26.

14. Brauer DG, Ferguson KJ. The integrated curriculum in medical education: AMEE Guide No. 96. Med Teach. 2015;37:312-22.

15. Ogur B, Hirsh D, Krupat E, Bor D. The Harvard Medical SchoolCambridge Integrated Clerkship: An innovative model of clinical educational. Acad Med. 2007;82(4):397-404.

16. Hansen L, Simanton E. Comparison of third-year student performance in a twelve-month longitudinal ambulatory program with performance in traditional clerkship curriculum. S D Med. 2009;62:315-7.

17. Poncelet A, Bokser S, Calton B, et al. Development of a longitudinal integrated clerkship at an academic health center. Med Educ Online. 2011;16:5939. https://doi.org/10.3402/meo.v16i0.5939

18. Dube B, Verduin M. A brief examination of integrated care in undergraduate medical education. Acad Psychiatry. 2015;39:457-60.

19. Madras BK, Compton WM, Avula D, Steqbauer T, Stein JB, Clark HW. Screening, brief interventions, referral to treatment (SBIRT) for illicit drug and alcohol use at multiple healthcare sites: comparison at intake and 6 months later. Drug Alcohol Depend. 2009;99(1-3):280-95.

APPENDIX

Table 2 Biopsychosocial Approach to Health Clerkship "Top Ten" Integrated Didactic Series

\begin{tabular}{ll}
\hline \hline Topic & Pedagogy \\
\hline $\begin{array}{l}\text { 1. Primary Care of the Psychiatric Patient } \\
\text { 2. Alcohol and SBIRT* }\end{array}$ & $\begin{array}{l}\text { Team-based learning } \\
\text { Role-play/skills workshop } \\
\text { 3. Tobacco Use and Cessation }\end{array}$ \\
$\begin{array}{l}\text { Case conference } \\
\text { 4. Decision-Making Capacity }\end{array}$ & $\begin{array}{l}\text { Team-based learning } \\
\text { 5. Chronic Pain I: CBT }{ }^{\dagger} \text { and Non- }\end{array}$ \\
$\quad$ Pharmacologic Management & \\
6. Chronic Pain II: Opioid Prescribing & Case conference \\
$\quad$ and Misuse & \\
7. Anxiety Disorders & Case conference \\
8. Adult and Pediatric Obesity & Team-based learning \\
9. Medically Unexplained Symptoms & Case conference \\
10. Reflective Writing in Medicine & Skills workshop \\
\hline
\end{tabular}

*SBIRT = screening, brief intervention, referral to treatment ${ }^{19}$

${ }^{+} \mathrm{CBT}=$ cognitive behavioral therapy 